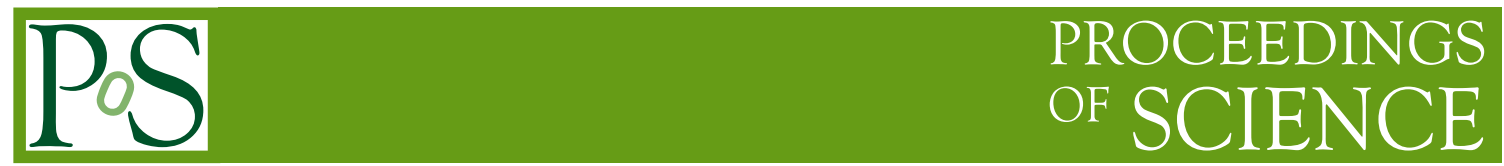

\title{
Glueballs from the Lattice
}

\section{Biagio Lucini* ${ }^{*}$}

Physics Department, Swansea University, Singleton Park, Swansea SA2 8PP, UK

E-mail: B.Lucini@swansea.ac.uk

Recent numerical calculations of the glueball spectrum in QCD, in $\mathrm{SU}(N)$ Yang-Mills theory in the large- $N$ limit and in candidate theories of strongly interacting dynamics beyond the standard model (in which the lowest-lying scalar plays the role of the Higgs boson) are reviewed and their implications for our theoretical understanding of glueballs in QCD-like theories and in strongly coupled gauge theories with a (near-)conformal dynamics are discussed.

From quarks and gluons to hadronic matter: A bridge too far?

2-6 September, 2013

European Centre for Theoretical Studies in Nuclear Physics and Related Areas (ECT*), Villazzano, Trento (Italy)

\footnotetext{
*Speaker.

${ }^{\dagger}$ In memory of Pierre van Baal, who greatly contributed to Lattice Gauge Theories.
} 


\section{Introduction}

One of the implications of colour confinement in $\mathrm{SU}(N)$ Yang-Mills theories is the existence of massive gauge-invariant colour singlet states with purely gluonic content, the glueballs. The coupling being of order one, a quantitative understanding of the glueball spectrum transcend perturbative techniques and can only be achieved in an ab-initio framework. Among $\mathrm{SU}(N)$ gauge groups, phenomenologically, a special role is played by SU(3), which is the gauge group of QCD. One expects that glueball states also appear in QCD. However, to date, glueballs have eluded any experimental attempt aimed at their identification in the QCD spectrum (see e.g. [1] for a recent review of the theoretical and experimental status of this field). The fundamental reason for the lack of experimental evidence of glueballs in the spectrum can be traced back to the fact that there is no quantum number that distinguishes glueballs from isosinglet mesons in the same $J^{P C}$ channel. Hence, physical states could naturally be thought of as admixtures of states that in idealised conditions one would call glueballs and states that are naturally identified as isosinglet mesons. This mixing can be assumed to be the result of some off-diagonal effective Hamiltonian that couples nearby states in the spectrum with the same quantum numbers. If the mixing is small, then one could classify the states that are coupled by this Hamiltonian under gluon-rich and quark-rich. However, it is a dynamical problem to establish the strength of the mixing terms.

As for other non-perturbative features of QCD, numerical calculations with Monte Carlo methods in the theory discretised on a spacetime lattice provide invaluable insights on the nature of glueballs (see e.g. [2] for a recent review and [3] and references therein for more recent developments). Those calculations should measure the effective couplings for the production and annihilation of glueballs and of mesons (which will be related to the diagonal elements of the effective Hamiltonian describing the interactions of those states) as well as the couplings between glueballs and mesons. Although progress in this direction has been achieved, a calculation of this type is still unviable. Thus, most of current lattice calculations assume that the mixing is small and compute masses of mesons and glueballs separately, the hope being that the real interacting spectrum will not look much different from this simplified case. In addition, since glueball measurements often demand a large statistics, which in turn requires a huge computational effort, the glueball spectrum is often provided in the quenched approximation, in this case amounting to studying the problem in the SU(3) Yang-Mills theory. The reference calculation in this approximation is still provided by [4], with some subsequent work [5] confirming the main results and providing some extra insights. The quenched approximation, which at first sight looks quite drastic, can in fact be formulated in a framework that provides a controlled interpolation from QCD to SU(3) Yang-Mills: the 't Hooft large- $N$ limit [6] (see [7] for an early pedagogical review of the main results).

In addition to the theoretical and experimental interest for glueballs in QCD, more recently there has been an increasing interest in glueball-like states in theories that like QCD confine in the infrared and are asymptotically free in the ultraviolet, but unlike QCD have an intermediate energy region in which the physics is governed by an infrared fixed point in an enlarged parameter space. These theories, normally referred as technicolour, are said to be walking, since the coupling runs very slow in this intermediate energy range ${ }^{1}$. Walking, and more in general infrared conformal

\footnotetext{
${ }^{1}$ Although the coupling is not a physical quantity, the concept of running can be formalised in terms of spectral observables.
} 
gauge theories, can provide a dynamical mechanism of electroweak symmetry breaking. A full discussion of this mechanism goes beyond the scope of this work, and we refer the interested reader to the specialised literature (e.g. [8] and references therein). Our interest here is in the fact that in this framework the lowest-lying isosinglet scalar plays a special role, since it should be identified with the recently discovered Higgs boson. Once again, the existence of such a light scalar is a dynamical problem that can be addressed with lattice calculations.

In this contribution, we give a brief overview of recent lattice results. This work does not aim at providing a comprehensive review, nor does it pretend to be complete. The main purpose here is to discuss some of the most recent results for glueball masses obtained from lattice calculations and their possible implications for our understanding of glueballs. A more detailed account of the field is provided in the quoted references and in works cited therein. The rest of this paper is structured as follows. In Sect. 2 we briefly review the lattice formulation of $\mathrm{SU}(N)$ gauge theories and of QCD, with a particular emphasis on widely employed techniques to extract glueball masses. Sect. 3 discusses numerical results in SU $(N)$ Yang-Mills and their relevance for QCD, providing also an explicit example of a QCD calculation. Calculations of the glueball spectrum in theories potentially relevant for explaining dynamically electroweak symmetry breaking are reported in Sect. 4. Finally, Sect. 5 summarises the main lessons learnt from those calculations and points out some of the current and future directions of lattice calculations of glueball properties.

\section{Extracting glueball masses from lattice calculations}

The lattice is the most reliable method for obtaining ab-initio quantitative predictions in the non-perturbative regime of QCD. The calculation starts with the QCD action regularised on a fourdimensional spacetime grid in such a way that gauge invariance is preserved. In the pure gauge sector, the simplest choice is the Wilson action:

$$
S_{w}=\beta \sum_{i, \mu>v}\left(1-\frac{1}{N} \mathscr{R} e \operatorname{Tr} U_{\mu v}(i)\right) .
$$

In the previous equation, $i$ labels a point in the discretised spacetime, $\mu$ and $v$ are two directions, and the sum extends over all points and directions. $\mathscr{R} e \operatorname{Tr} U_{\mu \nu}$ is the real part of the trace of the plaquette $U_{\mu v}$, with the latter being the path ordered product of the oriented link variables $U_{\mu}(i)$ (with reverse orientation corresponding to Hermitian conjugation) around an elementary square of the lattice. Positive orientations of the links connecting nearest neighbour sites follow the direction of the versors $\hat{\mu}$. A link variable $U_{\mu}(i)$ is an element of $\mathrm{SU}(3)$ (or of the gauge group, in the most general case), related to the continuum field $A_{\mu}$ by

$$
U_{\mu}(i)=\operatorname{Pexp}\left(i g a \int_{i}^{i+\hat{\mu}} A_{\mu}(x) \mathrm{d} x\right)
$$

with the integral taken from point $i$ to its nearest neighbour $i+\hat{\mu}$ along the direction $\hat{\mu}$ and $a$ being the lattice spacing. The lattice coupling $\beta$ is related to the continuum (bare) coupling $g$ by $\beta=2 N / g^{2}$, with $N$ the number of colours (i.e. $N=3$ for QCD). Gauge transformations are represented by functions $G(i)$ defined on the lattice points and taking values in the gauge group. They act on link variables by sending $U_{\mu}(i)$ to $G(i)^{\dagger} U_{\mu}(i) G(i+\hat{\mu})$, where the $\dagger$ symbol indicates 
Hermitian conjugation. It is straightforward to see that with these definitions the action $S_{w}$ is gauge invariant. It is also easy to see that, in the limit $a \rightarrow 0$, at the lowest order in $a, S_{w}$ reproduces the Yang-Mills continuum action. Using asymptotic freedom, it is possible to show that quantum fluctuations do not spoil the correctness of the continuum limit.

For fermions, on the lattice one can not preserve at the same time chiral symmetry and ultralocality of the action without generating spurious copies called doublers. Hence, if we want to have an action coupling a finite number of neighbour points, we have to give up either the possibility of having a generic number of flavours or exact chiral symmetry. Chirally symmetric fermionic actions with an arbitrary number of flavours can also be formulated, at the expenses of coupling all the sites (or, equivalently, of adding an extra dimension). It is worth stressing that (as one would expect) all lattice fermionic actions reproduce the same continuum physics, but some of them can be used more proficiently than others in particular physical situations. Without going further into specialistic technical details, the actions describing the fermion-gauge interactions we are interested in can be written as quadratic forms in terms of the fermion fields:

$$
S_{f}=\bar{\psi}_{i} M_{i j} \psi_{j}
$$

where $i$ and $j$ are Euclidean indices, with all other indices kept implicit. $M$ embodies the spinorial structure and depends linearly on the gauge fields $U_{\mu}$. The path integral of the full theory is

$$
Z=\int \mathscr{D} U_{\mu}(\operatorname{Det} M)^{N_{f}} e^{-S_{w}},
$$

where $N_{f}$ is the number of flavours, $\mathscr{D} U_{\mu}$ is the path integral measure of all links (which on the lattice is well defined and corresponds to a product of Haar measures) and the determinant of $M$ comes after the Grasmann integral over the fermions. Eq. (2.4) is amenable to Monte Carlo numerical simulations. State of the art numerical results require modern supercomputers.

Using the path integral (2.4), the vacuum expectation value of an operator $\mathscr{O}$ depending only on the link variables is obtained as

$$
\langle\mathscr{O}\rangle=\frac{1}{Z} \int \mathscr{D} U_{\mu}(\operatorname{Det} M)^{N_{f}} \mathscr{O} e^{-S_{w}} .
$$

For glueball mass calculations, $\mathscr{O}$ tales the form of a correlator of an appropriate observable $\bar{O}$ with the quantum numbers of the state of interest. If $\bar{O}$ depends on the point $i=(\vec{x}, t)$, where we have conventionally separated the Euclidean time coordinate $t$ and the space coordinates $\vec{x}$, we define $O(t)$, the zero-momentum operator at timeslice $t$, as

$$
O(t)=\frac{1}{V_{s}} \sum_{\vec{x}} \bar{O}(x, t)
$$

with $V_{s}$ the spatial volume. The correlator of interest is $C(\tau)=\left\langle O^{\dagger}\left(t_{0}\right) O\left(t_{0}+\tau\right)\right\rangle$, which, by time translational invariance, is a function of $\tau$ only. Using the spectral representation,

$$
C(\tau)=\int \mathscr{C}(E) e^{-E \tau} \mathrm{d} E
$$

At the lowest end of the spectrum, the spectral function $\mathscr{C}(E)$ is a discrete sum over Hamiltonian eigenstates with eigenvalues corresponding to physical masses of states contributing to $C(\tau)$. In 
the limit $\tau \rightarrow \infty$, only the lowest state with the quantum number of $\bar{O}(x, t)$ contributes to $\mathscr{C}(\tau)$. If $\bar{O}(x, t)$ is taken to transform under an irreducible representation of the rotational group, its energy will be the lowest mass $m_{J}$ in the channel with spin corresponding to that irreducible representation:

$$
m_{J}=-\lim _{\tau \rightarrow \infty} \frac{C(\tau)}{\tau}
$$

Since the lattice has a finite extent, the previous formula can be reliably used to extract the mass of glueballs as long as $\bar{O}$ is chosen in such a way that the contribution of higher excitations is minimised. An optimal choice of $\bar{O}$ can be performed only if the Hamiltonian eigenstates are known, but these are exactly what we are after. However, we can approximate these eigenstates using a variational method. In practice, we use a large set of operators $\bar{O}_{i}$ by considering traced path ordered products of link variables over closed contours of various shapes and sizes. Each fixed contour is transformed under the rotational group and the results are combined in such a way that irreducible representations are constructed. Using paths transformed under the appropriate symmetries, we build combinations that have also well defined parity and charge conjugations.

The same base paths can give an increased basis of operators by replacing the links with more extended objects. These new variables, often referred to as fat links, are still defined on lattice links, with a modification of the original link variables that accounts also for contributions coming from a neighbourhood of each link. The new links transform under gauge transformations as the original ones. The physical idea behind this procedure is that hadrons are extended objects, while the links are ultraviolet quantities. If we want to access the properties of physical particles we need to use variables that have support on the typical physical length. In practice, one replaces original variables with blocked variables, as in a block-spin transformation in statistical mechanics. These blocking transformations can be combined and iterated. There are various ways of implementing this idea. The results we review in this work follow Refs. [9, 10].

Having built a set of loops with well defined $J^{P C}$ transformation laws, we consider all possible correlators $C_{i j}(\tau)=\left\langle O_{i}^{\dagger}\left(t_{0}\right) O_{j}\left(t_{0}+\tau\right)\right\rangle$, where each operator is the trace of the ordered product over some fixed base loop of either the original links or the link variables obtained after a given number of iterations of the blocking transformations. The correlation matrix $C_{i j}(\tau)$ (normalised so that $C_{i j}(0)$ is the identity) is then diagonalised for a fixed $\tau$ (generally, $\tau=1$; although a higher $\tau$ would be desirable, rarely the statistics is sufficient to get an accurate signal for $\tau>1$ ). This procedure defines the eigenstates $\Phi_{1}(1), \ldots, \Phi_{M}(1)$, where $M$ is the dimension of $C$. For simplicity, we consider a case in which the eigenvalues are non-degenerate, the more general case only posing technical complications that could be dealt with quite easily. The eigenvectors are assumed to be ordered from the one corresponding to the higher to the one corresponding to the lower eigenvalue. Applying Eq. (2.8) to $\Phi_{k}$, one obtains a variational estimate of the mass for the excitation $k-1$ with quantum numbers $J^{P C}$.

In practice, things are more complicated. For a start, only a few low states are below the onset of a continuum spectral density, which starts with the multi-particle bound state of lowest possible energy. This requires us to identify scattering states and to project them out from our basis. Second, the spatial lattice has not the full rotational symmetry, but has the symmetry of the invariance group of the three-dimensional cube, the octahedral group, which has a finite number of elements (24 excluding parity). Hence, one needs to classify the operators according to the transformations of 
the octahedral group and then decompose the latter according to the continuum spin contributions, with more than one continuum spin contributing to each irreducible representation of the octahedral group. Then, on a finite lattice, there are spurious contributions to the glueball spectrum related to operators winding around the periodic spatial directions. These contributions, called torelon states, need to be removed from the spectrum. Finally, one has to keep in mind that the calculation is variational. While this is not a big problem for the lowest-lying states, the variational estimate of higher excitations is less and less accurate, since the number of operators in the variational basis decreases as we go higher in mass. These issues have been known since the early days of glueball calculations on the lattice (see e.g. [11]). Various approaches have been developed over the years, and glueball calculations for the ground state and a few excitations in some spin channels (e.g. the $0^{++}$and the $2^{++}$) are already quite accurate, at least in the pure Yang-Mills case. Specific calculations have targeted other quantum states, providing a rather comprehensive set of results, as for instance in $[4,5]$. Recently, the work [12] has appeared, in which the construction of the variational basis is fully automatised. This method allows us to perform a full calculation in all the channels, in which we can identify scattering and torelon contributions and hence remove them from the physical spectrum. This work - to which we refer for further details - is the foundation for the main results presented in the next section.

\section{From $\mathrm{SU}(3)$ to $\mathrm{SU}(\infty)$ and back to $\mathrm{QCD}$}

For a long time, performing calculations at quark masses that are low enough for being phenomenologically relevant has been a big practical problem in lattice QCD. Recent advances in numerical algorithms and computational power allow calculations to be done at a pseudoscalar mass close to that of the physical $\pi$. However, this still poses a computational challenge that limits the statistics. The number of configurations that can be obtained are generally sufficient for getting the meson spectrum at an acceptable accuracy level. However, numerical calculations of glueball masses require higher statistics (at least by a factor of ten) Monte Carlo simulations. The reason for the different statistical requirements to get an error of the same order of magnitude is well understood, and can be explained in terms of QCD dynamics. While the field is progressing at a fast pace, still the required computational time is highly non-trivial to obtain. Because of these high computational demands, it still makes sense to resort to the so-called quenched approximation, in which the quarks are treated as external sources. In glueball calculations of SU(3), this amounts to studying the pure Yang-Mills theory. At this stage, it is an open problem to establish whether and how these calculations could be relevant for real-world QCD.

In fact, a natural framework to interpolate from SU(3) to QCD, and hence to ascertain the importance of glueball calculations in pure gauge for the full dynamical theory, is provided by the 't Hooft large- $N$ limit. In this context, pure $\mathrm{SU}(3)$ gauge theory is seen as a particular case of the generic family of $\mathrm{SU}(N)$ Yang-Mills theory. As the number of colours $N$ is sent to infinity and the coupling of the theory $g$ is sent to zero with the constraint that the 't Hoof coupling $\lambda=g^{2} N$ remains constant, at the perturbative level the theory simplifies, and one can prove that observables can be expressed in a power series in $1 / N^{2}$. If the coefficients of this series are of order one, one would expect the physics in $\mathrm{SU}(3)$ to only differ from that of the large- $N$ extrapolated theory by a quantity of the order of $10 \%$ in each observable. 


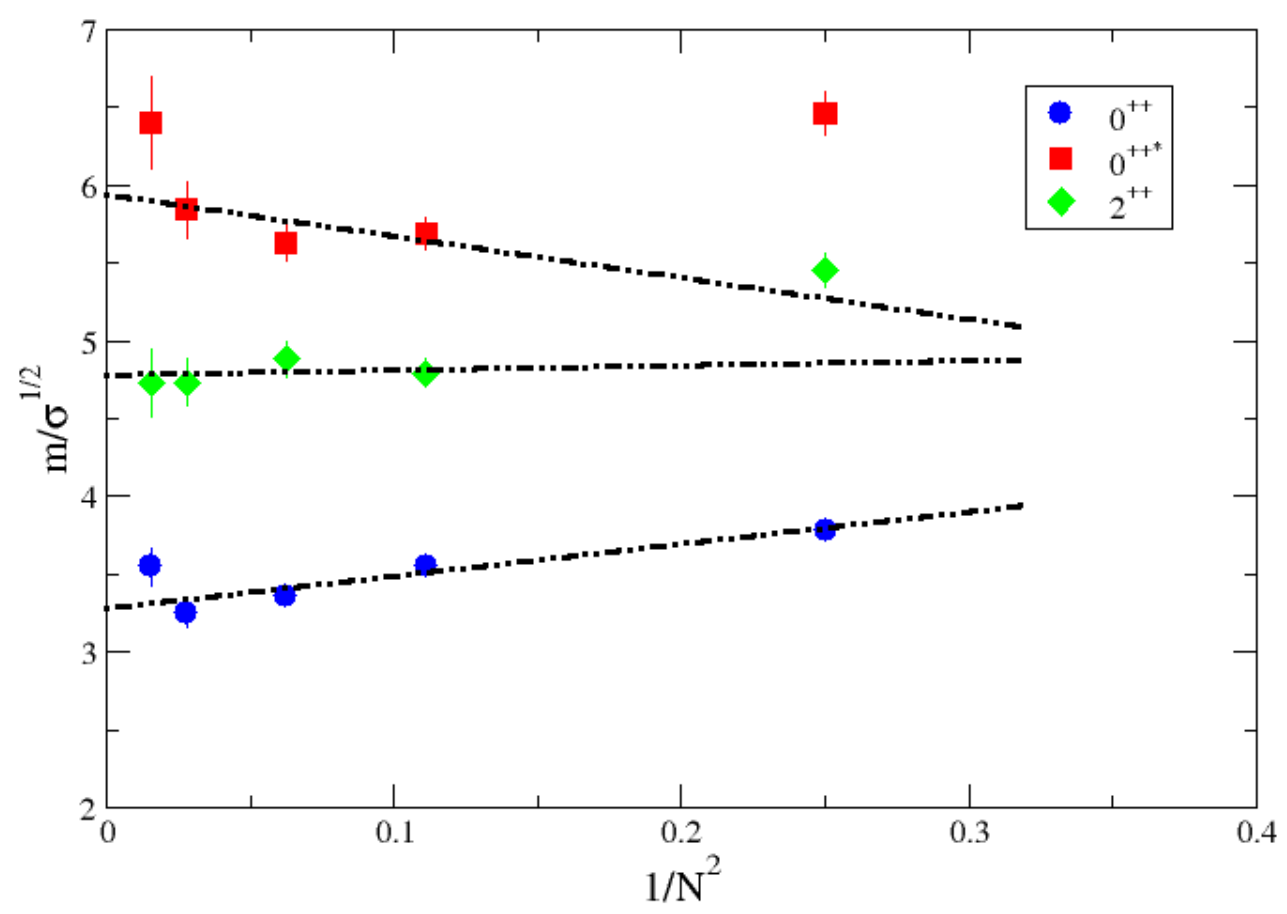

Figure 1: Extrapolations to $N \rightarrow \infty$ of the lowest-lying glueball masses.

Numerically, the determination of the large- $N$ limit of $\mathrm{SU}(N)$ gauge theories can be achieved by studying the values of observables for various $\mathrm{SU}(N)$ group and assuming the validity of the diagrammatic argument that suggests a power series in $1 / N^{2}$ [13]. For various observables, this procedure suggests that at the level of accuracy of a few percents the SU(3) value and the infinite$N$ value can be related using only the leading correction (see [14] and references therein).

The most precise determination of the $\mathrm{SU}(N)$ continuum limit of the lowest-lying glueball masses has been provided in [9] (see Fig. 1). Using the square root of the string tension $\sqrt{\sigma}$ to set the scale, the quoted results for the lowest mass in the $0^{++}$channel, the lowest mass in the $2^{++}$ channel and the first $0^{++}$excitation are respectively

$$
\begin{aligned}
\frac{m_{0^{++}}}{\sqrt{\sigma}} & =3.28(8)+\frac{2.1(1.1)}{N^{2}}, \\
\frac{m_{2^{++}}}{\sqrt{\sigma}} & =4.78(14)+\frac{0.3(1.7)}{N^{2}}, \\
\frac{m_{0^{++*}}}{\sqrt{\sigma}} & =5.93(17)-\frac{2.7(2.0)}{N^{2}} .
\end{aligned}
$$

The emerging picture is that, at level of accuracy of around 5-10\%, glueballs are well described by their large- $N$ limit, the correction coefficient being of order one. This is consistent with the expectation of the large- $N$ series being convergent at $N=3$. In this sense, and following the 


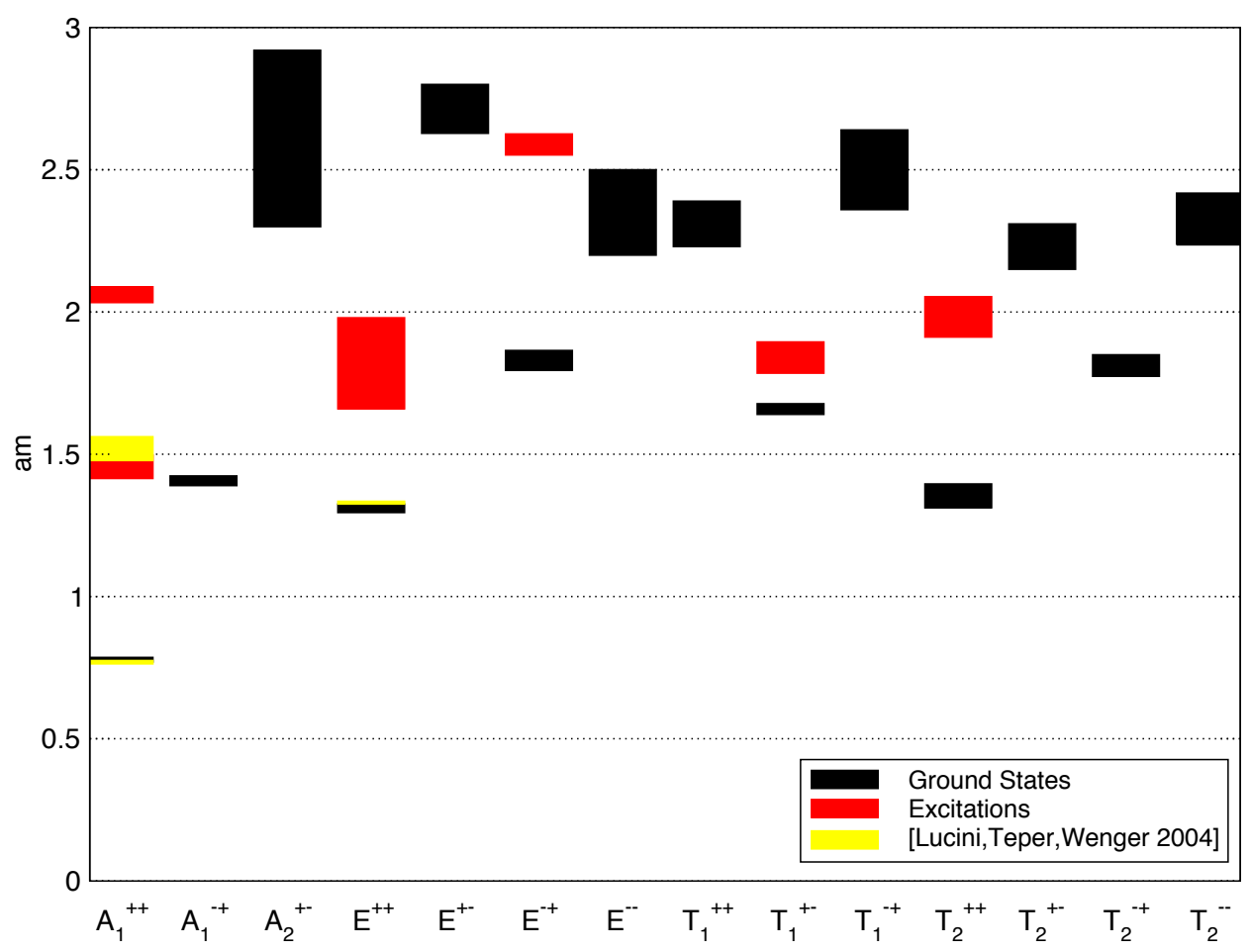

Figure 2: Glueball masses in various channels (ground states and some excitations) in the large- $N$ limit at fixed lattice spacing $a=1 /\left(6 T_{c}\right), T_{c}$ being the deconfinement temperature.

fact that the corrections are of the order of magnitude one would naively expect, one can say that $\mathrm{SU}(3)$ is close to $\mathrm{SU}(\infty)$. Hence, it makes sense to look at the physics of glueballs in the simpler $N=\infty$ theory. A calculation performed in [12] shows - albeit at fixed lattice spacing - that similar conclusions seem also to be valid for all glueball states currently accessible to numerical simulations (Fig. 2). This is good news for large- $N$ based approaches to glueballs and QCD-like strong interactions, like for instance the gauge-string duality (see e.g. [15] and references therein) or, more recently, topological field theory [16].

When dynamical fermions are considered, it can be shown that they provide a leading contribution that is $1 / N$-suppressed with respect to the Yang-Mills leading term. Hence, if SU(3) is close to $\mathrm{SU}(\infty)$ and the contribution given by quarks stays negligible for $N=3$ (as recent lattice calculations seem to confirm [17]), then glueball calculations in SU(3) gauge theory can still provide a useful guidance to experiments. This provides a motivation to perform high precision quenched calculations.

Another relevant consequence of the large- $N$ limit is that mesons and glueballs do not mix for infinite number of colours. If $\mathrm{SU}(3)$ is close to the large- $N$ limit, the suppression of mixing at large- $N$ can justify an approach to glueballs that neglects their mixing with mesons. Very recently, high statistics numerical calculations of the glueball spectrum in a full QCD setup have been per- 


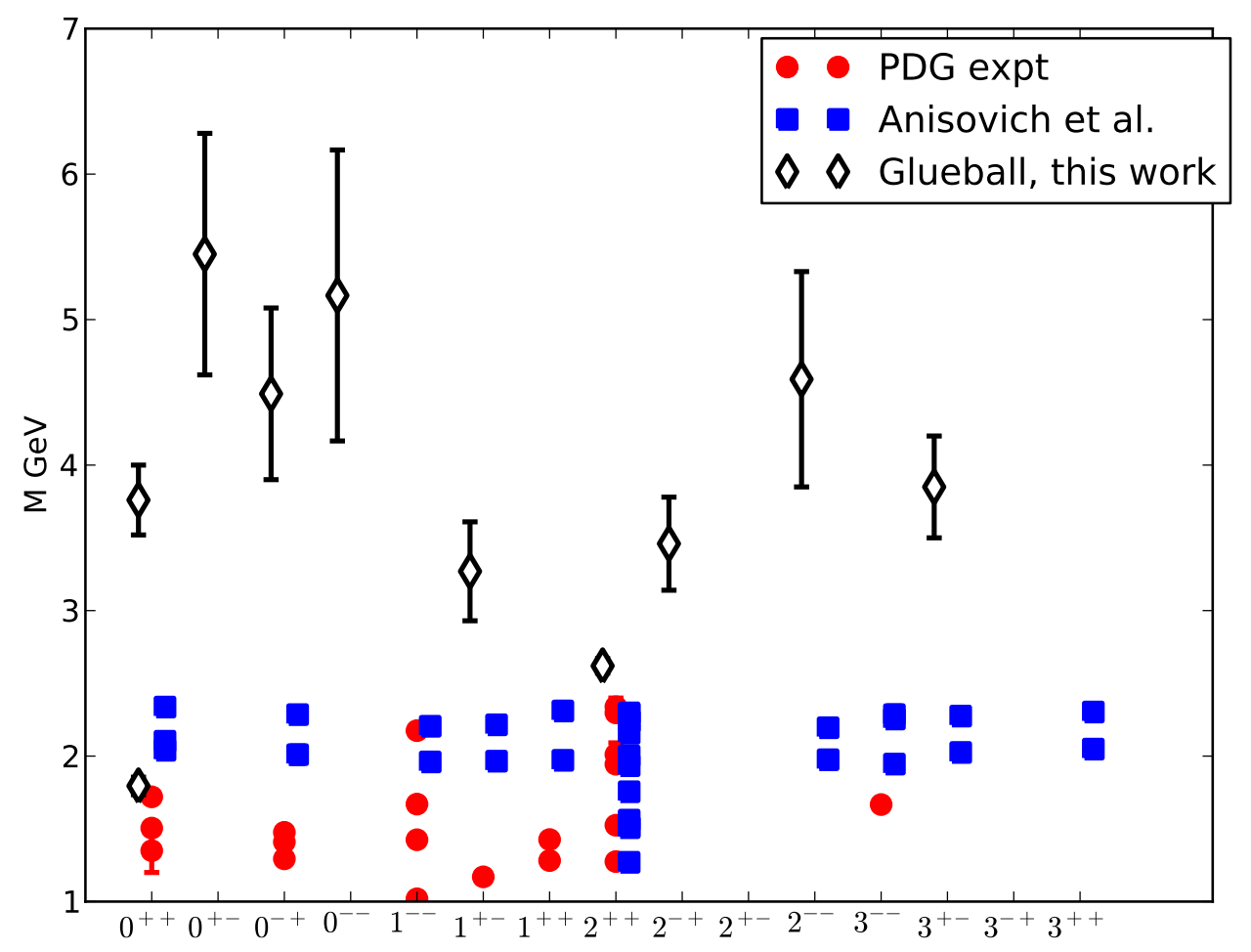

Figure 3: Glueball masses in full QCD from a lattice calculation with dynamical fermions (open points) compared to experimental results, as described in [3], from which the plot has been taken.

formed in [18] and extended to include more spectral states in [3]. The central results of the latter calculation are shown in Fig. 3, which compares the results of the simulations (open points) with experimental states. Most of the glueball states seem to be at quite high mass, in a region that will be accessible to the PANDA experiment [19]. A current focus of lattice calculations is to reduce both the statistical error and the systematic error (e.g. by taking into account mixing with mesons and improving spin identification). In particular, lattice calculations are focusing on exotic quantum numbers (as for instance $2^{+-}$and $0^{-+}$), since these can not be explained by the simple quark model.

\section{Glueballs in near-conformal gauge theories}

QCD provides an exemplar realisation of confinement and chiral symmetry breaking with the physics determined only by one mass scale. However, it has been conjectured for a long time that the particular realisation of QCD is not the only possibility for having confinement in the deep infrared in asymptotically free non-Abelian gauge theories. Another possibility is that the theory be approximately infrared conformal. Infrared conformal theories are realised for a number of fermions close to the point at which the system ceases to be asymptotically free. The range of 


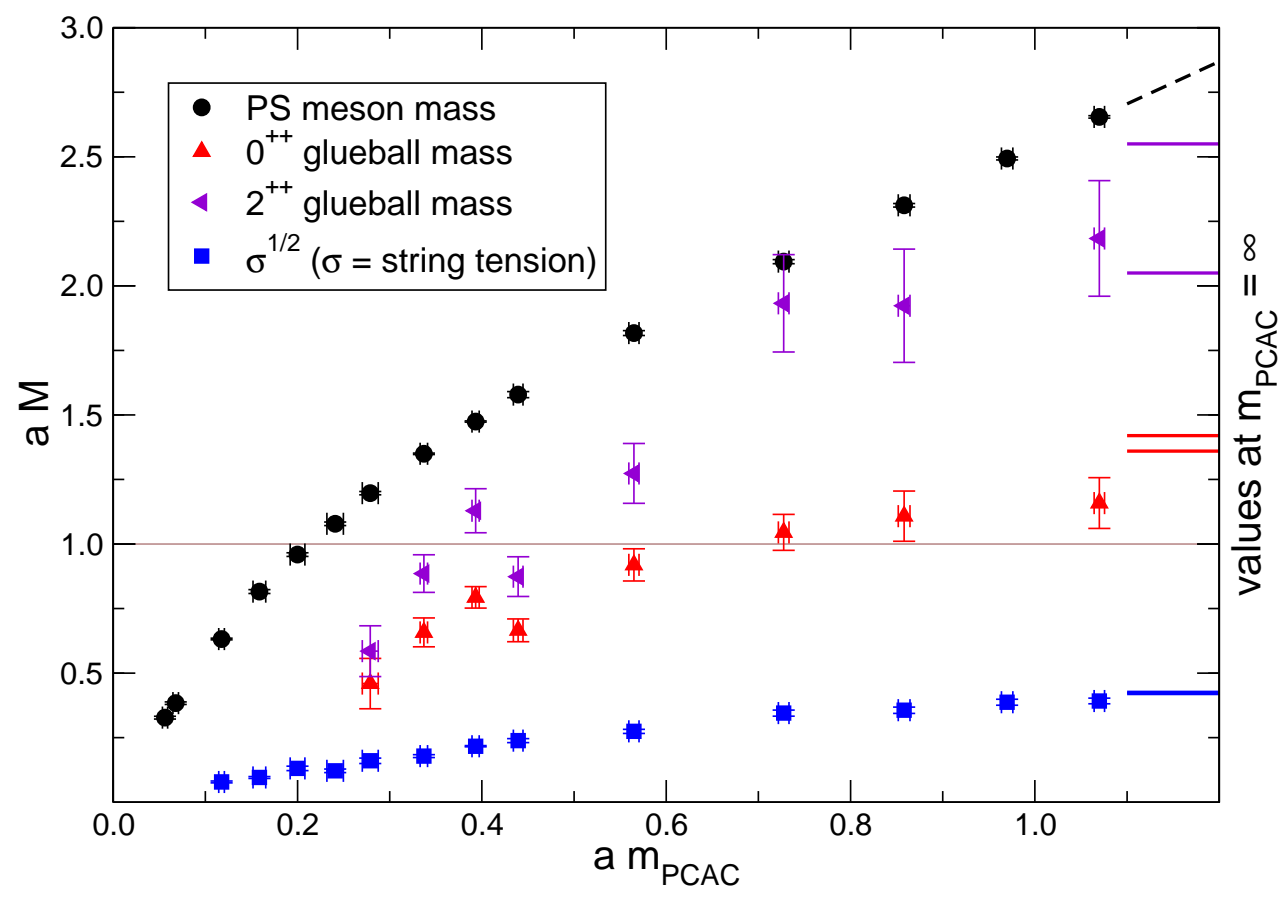

Figure 4: The lowest-lying states in the spectrum of SU(2) with two adjoint Dirac quarks [20], which has an infrared fixed point.

number of flavours for which the theory is infrared conformal is called conformal window. For theories just below the onset of the conformal window the infrared fixed point is only approximate, and for a wide range of energies observables have a mild dependence on the energy. Theories in this regime are said to be walking or near-conformal. A walking or a conformal novel interaction could provide a dynamical explanation of electroweak symmetry breaking.

As with other strong interactions, the lattice approach can provide essential information on the physical properties of those theories. Because of their potential relevance for phenomenology beyond the standard model, recently those theories have been widely studied with numerical simulations. One of the very first objectives has been the determination of the conformal window, which allows to pin down some candidate models for phenomenology. As a fundamental step, one must be able to disentangle features of (near-)conformality from a typical confining behaviour when the fermions have a finite (albeit small) mass. One of the signatures of a conformal gauge theory is a spectrum whose mass ratios do not depend on the fermion mass, possibly presenting an inverted mass hierarchy (i.e., unlike in QCD, glueball masses can be lower than the pseudoscalar meson mass [20, 21], see e.g. Fig. 4). This feature is particularly important in the light of the recent discovery of the Higgs particle, which in this framework will be identified with a scalar that is anomalously light near a point in which scale invariance gets restored. Although we have not seen yet this mechanism at work in gauge theories that can have phenomenological relevance, there are examples in gauge-string duality [22] and in lattice gauge theories in a strongly coupled regime far from the continuum limit [23]. In particular, lattice gauge theories at strong coupling can provide useful insights on the structure of conformal or nearly-conformal gauge theories [24]. 


\section{Conclusions}

The experimental identification of glueballs is one of the most urgent open problems in QCD. Thanks to recent progress, lattice calculations are getting closer and closer to providing firm theoretical predictions for glueball masses in all possible channels. Further progress will include a quantification of the mixing with mesons, a systematic study of states with exotic quantum numbers and a more accurate reconstruction of the continuum spin from the lattice data. More recently, glueballs (or better, $0^{++}$isosinglet states with a non-negligible contribution coming from the pure glue dynamics) are starting to play a key role in understanding the dynamics of strongly-coupled conformal or near-conformal gauge theories and in their phenomenological signatures, with a light glueball spectrum (originally identified for one of those models in [20]) now starting to emerge as a key signature of those theories [21]. Whether electroweak symmetry breaking is described by a gauge theory in the near conformal-phase and the Higgs particle should be seen as a glueball of a new strong interaction is current the subject of a fervid theoretical and phenomenological investigation, with the lattice proving to be once more an invaluable quantitative tool.

\section{Acknowledgments}

The results reviewed here have been obtained in various collaborations with L. Del Debbio, E. Gregory, A. Irving, C. McNeile, A. Patella, C. Pica. A. Rago, E. Rinaldi, M. Teper and U. Wenger. A special thanks goes to Craig McNeile, Antonio Rago and Enrico Rinaldi for their constant and crucial involvement in more recent glueball calculations. This work has been partially supported by the Royal Society grant UF09003 and the STFC grant ST/G000506/1. We also thank High Perfomance Computing Wales for computational resources.

\section{References}

[1] V. Mathieu, N. Kochelev, and V. Vento, The Physics of Glueballs, Int. J. Mod. Phys. E18 (2009) 1-49, [arXiv:0810.4453].

[2] C. McNeile, Lattice status of gluonia/glueballs, Nucl.Phys.Proc.Suppl. 186 (2009) 264-267, [arXiv:0809.2561].

[3] E. Gregory, A. Irving, B. Lucini, C. McNeile, A. Rago, et al., Towards the glueball spectrum from unquenched lattice QCD, JHEP 1210 (2012) 170, [arXiv: 1208 . 1858].

[4] C. J. Morningstar and M. J. Peardon, The glueball spectrum from an anisotropic lattice study, Phys. Rev. D60 (1999) 034509, [hep-lat/99010 0 4].

[5] H. B. Meyer and M. J. Teper, Glueball Regge trajectories and the pomeron: A Lattice study, Phys.Lett. B605 (2005) 344-354, [hep-ph / 0409183 ]. Y. Chen, A. Alexandru, S. Dong, T. Draper, I. Horvath, et al., Glueball spectrum and matrix elements on anisotropic lattices, Phys.Rev. D73 (2006) 014516, [hep-lat/0510074].

[6] G. 't Hooft, A planar diagram theory for strong interactions, Nucl. Phys. B72 (1974) 461.

[7] S. R. Coleman, $1 / N$, .

[8] J. Andersen, O. Antipin, G. Azuelos, L. Del Debbio, E. Del Nobile, et al., Discovering Technicolor, Eur.Phys.J.Plus 126 (2011) 81, [arXiv: 1104 .1255]. 
[9] B. Lucini, M. Teper, and U. Wenger, Glueballs and k-strings in SU(N) gauge theories: Calculations with improved operators, JHEP 0406 (2004) 012, [hep-lat / 0404008 ].

[10] B. Lucini, The Large N limit from the lattice, Few Body Syst. 36 (2005) 161-166, [hep-ph/0410016].

[11] B. Berg and A. Billoire, Glueball Spectroscopy in Four-Dimensional SU(3) Lattice Gauge Theory. 1., Nucl.Phys. B221 (1983) 109. C. Michael and M. Teper, The Glueball Spectrum in SU(3), Nucl.Phys. B314 (1989) 347.

[12] B. Lucini, A. Rago, and E. Rinaldi, Glueball masses in the large N limit, JHEP 1008 (2010) 119, [arXiv:1007.3879].

[13] B. Lucini and M. Teper, $S U(N)$ gauge theories in four-dimensions: Exploring the approach to $N=$ infinity, JHEP 0106 (2001) 050, [hep-lat/ 0103027 ]. B. Lucini and M. Teper, Confining strings in $S U(N)$ gauge theories, Phys. Rev. D64 (2001) 105019, [hep-lat/ 0107007 ].

[14] B. Lucini and M. Panero, SU(N) gauge theories at large N, Phys.Rept. 526 (2013) 93-163, [arXiv:1210 . 4997]. B. Lucini and M. Panero, Introductory lectures to large-N QCD phenomenology and lattice results, arXiv:1309.3638.

[15] D. Elander, A. F. Faedo, C. Hoyos, D. Mateos, and M. Piai, Multiscale confining dynamics from holographic RG flows, arXiv:1312.7160.

[16] M. Bochicchio, Glueball and meson spectrum in large-N massless QCD, arXiv:1308.2925.

[17] G. S. Bali, F. Bursa, L. Castagnini, S. Collins, L. Del Debbio, et al., Mesons in large-N QCD, JHEP 1306 (2013) 071, [arXiv: 1304 . 4437]. G. S. Bali, L. Castagnini, B. Lucini, and M. Panero, Large-N mesons, arXiv:1311. 7559.

[18] UKQCD Collaboration, C. M. Richards, A. C. Irving, E. B. Gregory, and C. McNeile, Glueball mass measurements from improved staggered fermion simulations, Phys. Rev. D82 (2010) 034501, [arXiv:1005.2473].

[19] PANDA Collaboration Collaboration, M. Lutz et al., Physics Performance Report for PANDA: Strong Interaction Studies with Antiprotons, arXiv:0903.3905.

[20] L. Del Debbio, B. Lucini, A. Patella, C. Pica, and A. Rago, Conformal versus confining scenario in SU(2) with adjoint fermions, Phys.Rev. D80 (2009) 074507, [arXiv: 0907 . 3896 ]. L. Del Debbio, B. Lucini, A. Patella, C. Pica, and A. Rago, The infrared dynamics of Minimal Walking Technicolor, Phys.Rev. D82 (2010) 014510, [arXiv:1004.3206].

[21] Y. Aoki, T. Aoyama, M. Kurachi, T. Maskawa, K.-i. Nagai, et al., Light composite scalar in twelve-flavor QCD on the lattice, Phys.Rev.Lett. 111 (2013) 162001, [arXiv:1305.6006].

A. Athenodorou, E. Bennett, G. Bergner, B. Lucini, and A. Patella, First results for SU(2) Yang-Mills with one adjoint Dirac Fermion, arXiv:1311.4155.

[22] D. Elander and M. Piai, On the glueball spectrum of walking backgrounds from wrapped-D5 gravity duals, Nucl.Phys. B871 (2013) 164-180, [arXiv:1212.2600].

[23] B. Lucini, A. Patella, A. Rago, and E. Rinaldi, Infrared conformality and bulk critical points: SU(2) with heavy adjoint quarks, JHEP 1311 (2013) 106, [arXiv:1309.1614].

[24] P. de Forcrand, S. Kim, and W. Unger, Conformality in many-flavour lattice QCD at strong coupling, JHEP 1302 (2013) 051, [arXiv: 1208 . 2148]. E. Tomboulis, Absence of chiral symmetry breaking in multi-flavor strongly coupled lattice gauge theories, Phys.Rev. D87 (2013) 034513, [arXiv:1211.4842]. 